\title{
Chiari Type II malformation: past, present, and future
}

\author{
Kevin L. Stevenson, M.D. \\ Children's Healthcare of Atlanta, Atlanta, Georgia
}

\begin{abstract}
Object. The Chiari Type II malformation (CM II) is a unique hindbrain herniation found only in patients with myelomeningocele and is the leading cause of death in these individuals younger than 2 years of age. Several theories exist as to its embryological evolution and recently new theories are emerging as to its treatment and possible prevention. A thorough understanding of the embryology, anatomy, symptomatology, and surgical treatment is necessary to care optimally for children with myelomeningocele and prevent significant morbidity and mortality.

Methods. A review of the literature was used to summarize the clinically pertinent features of the CM II, with particular attention to pitfalls in diagnosis and surgical treatment.

Conclusions. Any child with CM II can present as a neurosurgical emergency. Expeditious and knowledgeable evaluation and prompt surgical decompression of the hindbrain can prevent serious morbidity and mortality in the patient with myelomeningocele, especially those younger than 2 years old. Symptomatic CM II in the older child often presents with more subtle findings but rarely in acute crisis. Understanding of CM II continues to change as innovative techniques are applied to this challenging patient population.
\end{abstract}

\section{KEY WORDS • Chiari Type II malformation • myelomeningocele • pediatric}

The CM II is uniquely associated with myelomeningocele and is found only in this population. Originally described by Hans Chiari in 1891, symptomatic CM II accounts for more deaths before the age of 2 years in patients with spinal dysraphism than any other cause. ${ }^{18}$ Symptoms range from very subtle to life-threatening, requiring astute and occasionally rapid decision making and surgical intervention to prevent significant morbidity and mortality. Despite advances in neuroimaging, symptomatic CM II is diagnosed clinically and a thorough understanding of this entity is mandatory for those who work with this challenging patient population. This article will review CM II from embryological development to the operating room, with particular attention to the pathoembryology, anatomy, and clinical presentation.

\section{HISTORICAL BACKGROUND}

The first published report of what would come to be known as the CM II appeared in $1891 .{ }^{6}$ Hans Chiari, Professor of Pathology at the German University in Prague, published a series on hindbrain herniations based on autopsy findings. Chiari divided his series of patients into

Abbreviations used in this paper: $\mathrm{CM}=$ Chiari malformation; $\mathrm{CNS}=$ central nervous system; $\mathrm{CSF}=$ cerebrospinal fluid; $\mathrm{MR}=$ magnetic resonance; PEAC $=$ prolonged expiratory apnea with cyanosis. four distinct forms of the malformation, including the Type II malformation that he found exclusively in patients with myelomeningocele. Originally described as caudal displacement of the vermis, fourth ventricle, and brainstem, the CM II was later found to be associated with abnormalities throughout the brain, skull, and spine.

Two contemporaries of Chiari deserve mention for their early contributions to the description of the CM II. In 1883 , Cleland ${ }^{7}$ published a report on a single infant with myelomeningocele and hindbrain abnormalities similar to Chiari's Type II malformation. Although published 8 years prior to Chiari's initial report, Cleland's work remained relatively unnoticed. Three years after the initial report by Chiari, Arnold ${ }^{1}$ published a report, again on a single patient, with myelomeningocele and herniation of the cerebellum into the cervical canal. Although both Cleland and Arnold contributed to the early body of literature on the subject, it is clearly Chiari who deserves to have his name associated with the malformation. Rather than describe a single patient, Chiari performed in-depth pathological analyses of the hindbrain abnormalities and broke them into four distinct subtypes, a classification system that has not significantly changed since his initial report more than 100 years ago. For this reason, the term Chiari Type II malformation is preferred over the term Arnold-Chiari malformation. The first "Chiari decompression" occurred in 1932 reported by Graftidijk. ${ }^{33}$ Of the handful of patients reported, none survived. 


\section{PATHOEMBRYOLOGY AND PATHOPHYSOLOGY}

Understanding the clinical complexities of CM II requires understanding the pathoembryology and pathophysiology involved in the development of the malformation. Although it would be tempting to describe the CMs I to IV as a spectrum of a similar process, each CM is distinctly different from the next. Although no single error in development appears to produce the anomaly, four main theories have been offered in an attempt to explain the diverse hindbrain pathology seen in the CM II.

In his initial theory, Chiari attributed the hindbrain herniation to hydrocephalus. ${ }^{6}$ Although hydrocephalus is clearly associated with myelomeningocele, and cerebellar vermian herniation has been induced by a CSF pressure differential in the cranial and spinal compartments, ${ }^{42}$ this theory fails to explain many of the features of the CM II. From prenatal imaging it is known that the CM II is often present prior to the radiographic appearance of hydroephalus. ${ }^{20}$ Moreover, 10 to $20 \%$ of children with myelomeningocele and CM II never develop hydrocephalus and do not require shunt placement. ${ }^{31}$ In addition, the small posterior fossa, low-lying torcular herophili, and upward "herniation" of the vermis are not explained by this theory.

Primary dysgenesis of the hindbrain, initially postulated by Cleland, ${ }^{7}$ helps to explain the CNS malformations in the posterior fossa but fails to account for the cranial and supratentorial anomalies frequently associated with CM II and myelomeningocele. Overcrowding of the posterior fossa due to a mesodermal disorder resulting in a low-volume posterior fossa has been suggested as the cause of vermian and brainstem herniation. ${ }^{15} \mathrm{~A}$ similar concept involves an "induced" small posterior fossa secondary to CSF chronically leaking from the open spinal defect. ${ }^{23}$ These theories again fail to explain the widespread CNS abnormalities seen in patients with myelomeningocele.

Traction theorists, including Penfield, proposed the development of CM II by the open and tethered spinal cord pulling the hindbrain caudally, which results in the vermian and brainstem herniation seen in CM II. ${ }^{26}$ Again failing to account for many of the CNS deformities, this theory is further flawed by the fact that traction forces applied at the lumbar spinal cord are essentially nonexistent beyond four spinal levels from the tethered site. ${ }^{11}$

Combining features of each of the four previously mentioned theories is McLone and Knepper's ${ }^{19}$ unified theory of CM II. In this theory, both the open neural tube defect and incomplete spinal occlusion allow CSF to drain through the central canal and it is therefore not maintained in the ventricular system. Ventricular distention is required to induce both neural and calvarial development and without this ventricular CSF driving force the posterior fossa never fully develops. With later rapid growth of the rhombencephalon, the cerebellum is forced both cephalad and caudad along with the brainstem. Polymicrogyria, enlargement of the massa intermedia, low-lying tentorium and torcular herophili, and base of skull anomalies are produced by the same lack of ventricular distention in the telencephalon. This theory also explains the development of hydrocephalus due to impaction of the posterior fossa contents on the foramen magnum; with CSF outflow blocked or impaired at the foramina of Luschka and Magendie progressive ventriculomegaly results.

\section{ANATOMY}

A CM II is universally present in every child born with myelomeningocele. Like the open neural tube defect, the anatomical disturbance is varied in degree from child to child. The cerebellar vermis is invariably displaced below the foramen magnum and takes on the appearance of a "peg" (Fig. 1). The vermis can be impressively low, occasionally entering the upper thoracic canal. Documented elongation of the cerebellar peg over time has been noted on MR imaging with the caudal tip of the displaced vermis becoming proportionately more distant from the foramen magnum as the child ages. ${ }^{32}$ Cerebellar "inversion" (upward herniation above the tentorium) is often noted with the cerebellum located lateral to the midbrain in the cerebellopontine angle. ${ }^{25}$ In addition to its abnormal location, the cerebellum as a whole is small and appears thin. The abnormal shape of the cerebellum combined with an absent cisterna magna produces the ultrasonographic "banana sign." This finding is better seen on prenatal ultrasonography than the neural tube defect itself and can aid in prenatal diagnosis. ${ }^{39}$ The cerebellar folia may be shallow to absent, especially in the caudal half and vermis, and heterotopias are common (Fig. 2). ${ }^{43}$ Despite the small size of the cerebellum, the posterior fossa appears "crowded" due the abnormally small size of the compartment. The tentorium, typically hypoplastic, inserts abnormally low often placing the torcular herophili just above the foramen magnum, a critical point to keep in mind when a suboccipital craniectomy is being considered or performed (Fig. 3).

A varying segment of the brainstem is also located

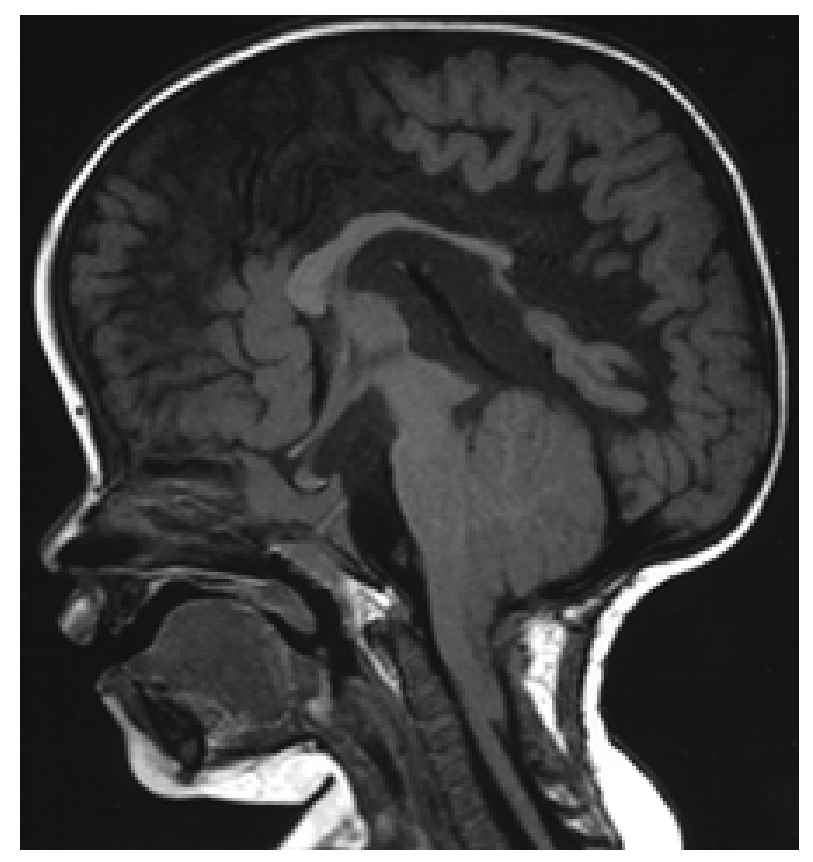

Fig. 1. Sagittal $\mathrm{T}_{1}$-weighted $\mathrm{MR}$ image revealing the peglike appearance of the vermis extending to $\mathrm{C}-4$ in a child with $\mathrm{CM}$ II. 


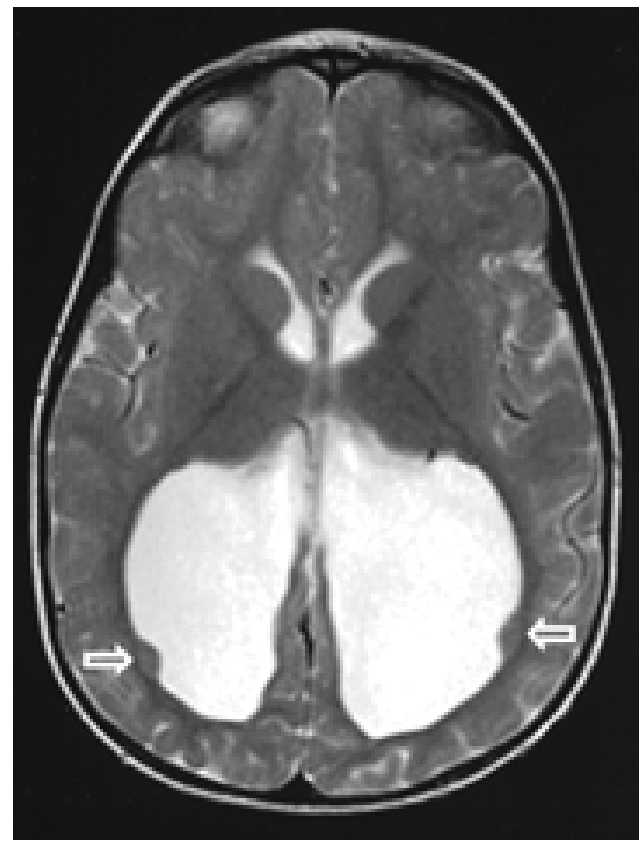

Fig. 2. Axial $\mathrm{T}_{2}$-weighted $\mathrm{MR}$ image obtained in a child with two subependymal heterotopias (open arrows). Note the marked culpocephaly despite a properly functioning shunt.

within the cervical canal, commonly the medulla but occasionally the lower pons as well. Approximately two thirds of patients will display a medullary "kink" dorsal to the upper cervical spinal cord (Fig. 4). ${ }^{9}$ The dorsal displace-

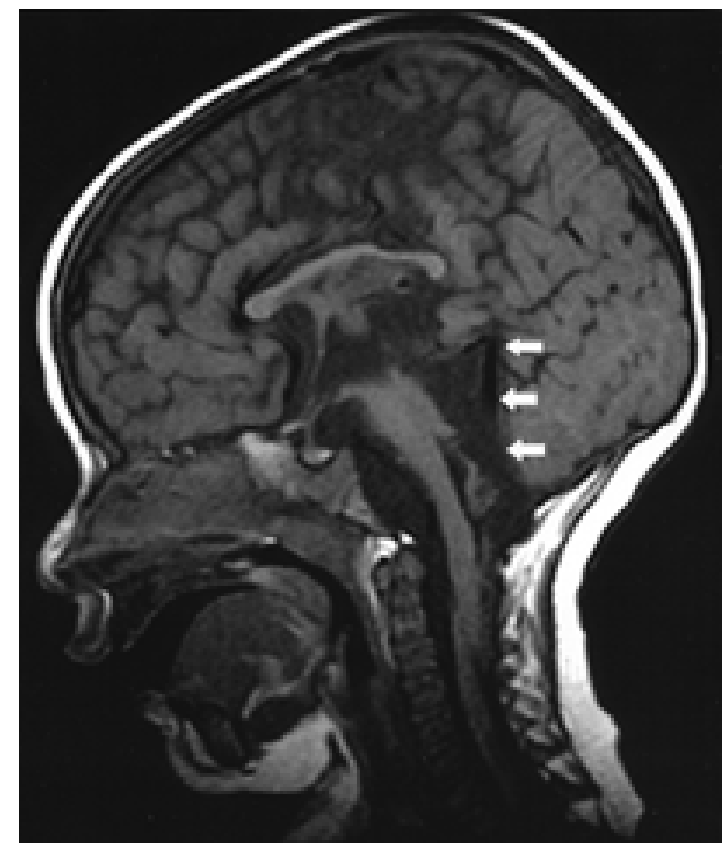

Fig. 3. Sagittal $\mathrm{T}_{1}$-weighted $\mathrm{MR}$ image obtained in a child with a CM II, revealing several classic findings. The tentorium (arrows) inserts abnormally low, placing the torcular herophili just inside the rim of the foramen magnum. Polygyria is also seen throughout the hemisphere.

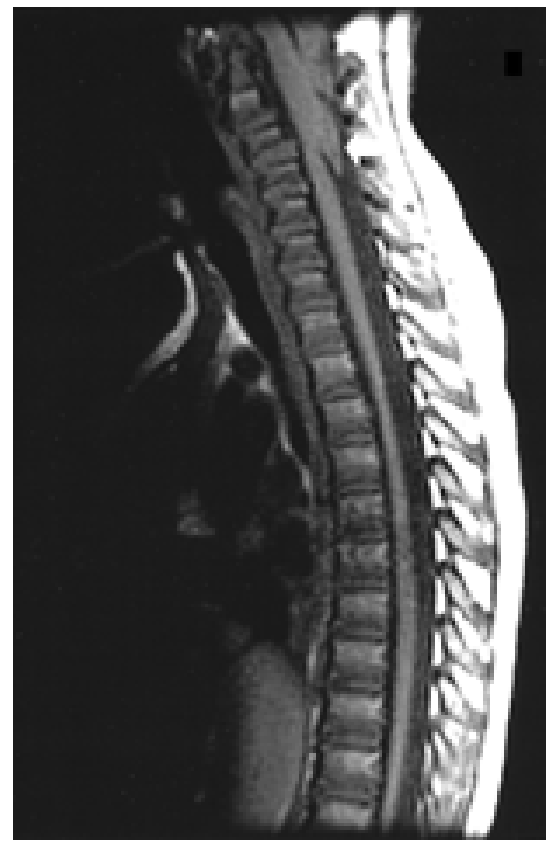

Fig. 4. Sagittal $\mathrm{T}_{1}$-weighted MR image of the cervicothoracic spine obtained in a child, revealing a severe medullary kink at C3-4 (arrow). Note that the cerebellar vermis ends cephalad to the kink.

ment of the relatively mobile medulla combined with the spinal cord being fixed by the dentate ligament produces this often dramatic finding. The presence of a medullary kink has been associated with a more symptomatic clinical course in $75 \%$ of patients with this finding below C-4 and the absence of clinical symptoms when the kink is either absent or located above C- $4 .{ }^{8}$ Depending on the presence and severity of the medullary kink, the lower cranial nerves exiting from the medulla can travel in a cephalad direction, and the tracts of the spinal cord can double back on themselves for a short distance. Tectal "beaking" can be noted due to fusion of the colliculi forming a single mass (Fig. 5), and the majority of patients display an enlarged massa intermedia (Fig. 6). ${ }^{9}$

Although histologically normal, the cerebral cortex in patients with CM II is abnormal in gross appearance. The gyri are abnormally numerous and small; however, the term polymicrogyria is to be avoided because of its association with an abnormal four-layered cortex that is not present in CM II; the term polygyria is preferred (Fig. 3). ${ }^{17}$ Partial-to-total agenesis of the corpus callosum is seen in $33 \%$ of patients with CM II; $62 \%$ of those have below average intelligence (Fig. 5). ${ }^{41}$

Multiple ventricular anomalies are commonly found in the patient with CM II. The fourth ventricle, which is typically small and poorly visualized, is frequently displaced into the cervical canal along with its choroid plexus. The aqueduct is similarly small and rarely seen on routine imaging, but does not likely contribute significantly to hydrocephalus. ${ }^{25}$ The third ventricle is rarely enlarged but may take on a narrow-angled appearance, giving rise to the term "shark tooth deformity." appearance varies from nearly normal to severely deformed and hydrocephalic. Colpocephaly is common with 


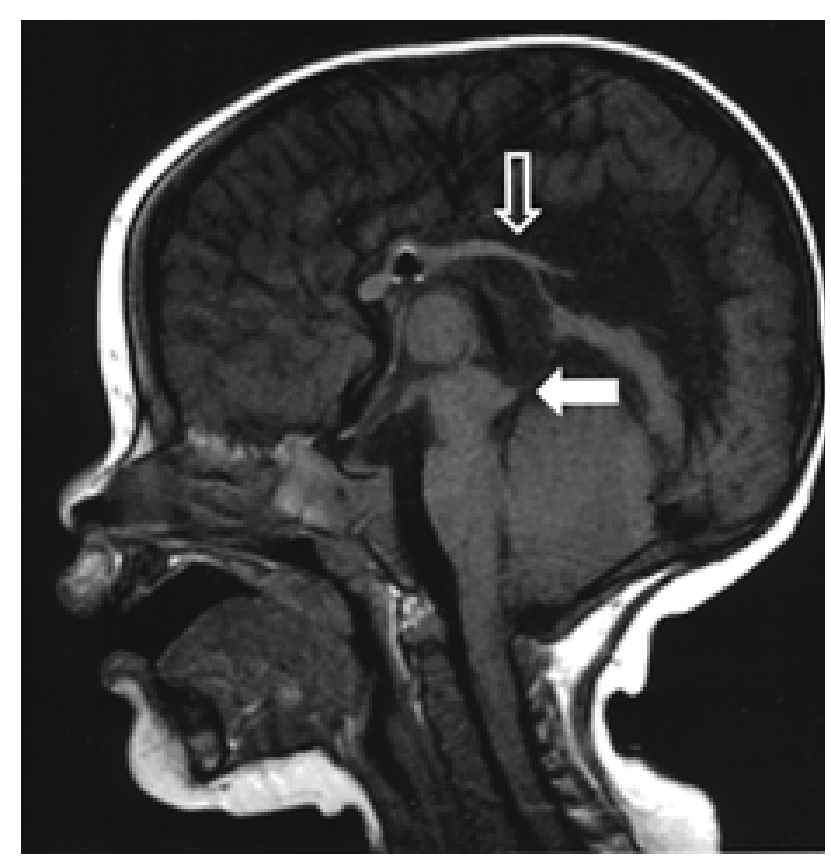

Fig. 5. Sagittal $\mathrm{T}_{1}$-weighted MR image obtained in a child with myelomeningocele and CM II. The tectum is beaked (closed arrow) and the corpus callosum shows partial agenesis (open arrow).

the occipital horns disproportionately enlarged compared with the frontal horns (Fig. 2). This finding is often present even in patients with myelomeningocele who do not have hydrocephalus and frequently persists in patients whom a shunt has been placed. "Beaking" of the frontal horns is occasionally seen when the frontal horns point

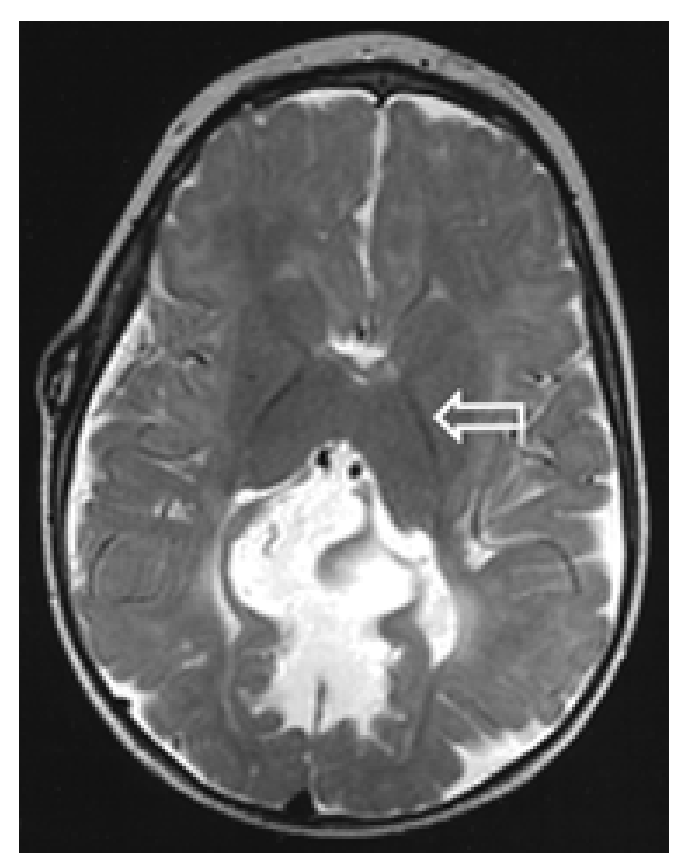

Fig. 6. Axial $\mathrm{T}_{2}$-weighted MR image obtained in a child with CM II and an enlarged massa intermedia (arrow). inferiorly. This finding is attributed to interdigitations of the cerebral hemispheres in the region..$^{30}$

In addition to the anomalies of the CNS, typical skull malformations are frequently found in association with CM II. The foramen magnum is often enlarged; this finding obviates the need for suboccipital craniectomy in many patients undergoing surgery for symptomatic CM II. Luckenschadel, scalloping of the petrous pyramid, and shortening of the clivus are common findings on computerized tomography scanning. ${ }^{21}$

\section{Signs and Symptoms}

Given that CM II is universally associated with myelomeningocele, the first sign is an open neural tube defect. Once born, a symptomatic CM II is the most common cause of death in patients with myelomeningocele who are younger than 2 years of age. ${ }^{18}$ Approximately one third of patients with CM II develop signs and symptoms of brainstem compression through the age of 5 years, and more than one third of these patients do not survive. ${ }^{14}$ Symptoms related to CM II vary markedly according to the age of the patient and can be broken into two groups: patients younger than the age of 2 years and those older than 2 years of age. Whatever the age of the child, the first consideration in evaluating symptomatic CM II must be hydrocephalus and the shunt, if present. Untreated hydrocephalus or a malfunctioning shunt can turn a radiographically evident CM II into a symptomatic CM II by increasing the intracranial pressure with subsequent downward herniation of an already caudally displaced brainstem and vermis. Ensuring that a shunt is working optimally or treating as yet untreated hydrocephalus is the first step in managing symptomatic CM II.

Infants and children younger than the age of 2 years present most frequently with cranial nerve and brainstem signs. Symptomatic children in this age group must be evaluated urgently, because a symptomatic CM II in this population can be a neurosurgical emergency. The most common, and potentially fatal symptom in this age group involves respiratory difficulties. Within this symptom complex, inspiratory stridor is often the most noticeable sign of brainstem dysfunction and should prompt urgent evaluation. Vocal cord abduction paresis or paralysis resulting from dysfunction of cranial nerve 10 produces the classic inspiratory stridor. Debate exists as to whether this cranial nerve dysfunction is due to traction on the nerve as it is pulled caudally by the herniated medulla/medullary kink, compression of the lower brainstem, ${ }^{27,28,32,40}$ or an inherently malformed dorsal motor nucleus within the brainstem. ${ }^{10,12,35}$ This debate impacts on clinical decision making when the child with myelomeningocele presents to the pediatrician or neurosurgeon with stridor. Although there clearly exists a spectrum of both cranial nerve nucleus dysgenesis and compression/traction, this sign must be evaluated urgently and not assumed to be due to a viral upper respiratory illness or other common pediatric airway illness. Direct laryngoscopy will confirm the diagnosis and should be performed on an emergency basis if symptomatic CM II is suspected. Another potentially lethal respiratory sign includes PEAC. When PEAC occurs it manifests as total cessation of expiratory effort with cyanosis that can lead to bradycardia and death, typically 
during a startling or painful experience. These events are not due to vocal cord paresis or paralysis; they can also occur in mechanically ventilated patients. More than one half of patients with CM II and apnea during infancy can be expected to die of PEAC, ${ }^{5}$ and therefore no infant with CM II should be assumed to be displaying typical breatholding of infancy.

Gastrointestinal disturbances are as prevalent as respiratory symptoms in patients with CM II, but they are more insidious in nature. Cranial nerves nine and 10 are frequently affected and manifest as neurogenic dysphagia. Chronic aspiration (leading to frequent pneumonia), choking, nasal regurgitation, prolonged feeding time, and weight loss are all symptoms of problematic CM II. Physical findings include coarse upper airway sounds, diminished-to-absent gag reflex, and emaciation. Barium swallow studies often show pharyngoesophageal dysmotility, cricopharyngeal achalasia, nasal regurgitation, and frank tracheal aspiration. Upper gastrointestinal evaluation is helpful prior to decompressive surgery as weight loss and poor nutritional status may lead to a higher incidence of postoperative complications; correcting these abnormalities prior to surgery, if time allows, will benefit the patient. Aggressive nasogastric feeding and/or funduplication can quickly reverse severe nutritional deficiencies and prepare the infant for surgery. Neurogenic dysphagia, which frequently appears prior to the onset of respiratory symptoms or other brainstem dysfunction, is nearly always progressive,$^{29}$ and should therefore be considered a harbinger of further neurological decline. Other signs and symptoms of CM II in infants include para(more commonly upper extremities) or quadriparesis, hypotonia, opisthotonos, nystagmus, weak cry, and developmental delay.

Unlike the case in infants, symptomatic CM II in older children is rarely a neurosurgical emergency. Symptoms in this age group tend to be more insidious and slowly progressive and are far less frequently life-threatening. Signs and symptoms of cervical myelopathy are the hallmark finding in this age group, with upper-extremity weakness and spasticity being the most common finding. Changes in handwriting skills, dexterity, and self-care may be the first outwardly noticeable signs. Ataxia, of both the upper extremities and trunk, is also common. Occipital headache and/or craniocervical pain, similar to that experienced in the CM I, can be present in the absence of more serious neurological symptoms. Syringomyelia is associated with CM II as in CM I and should be searched for in the symptomatic child (Fig. 7). Suspended disassociated sensory loss, hand atrophy, scoliosis, back pain, and lower motor neuron findings should prompt radiological evaluation of the spine, specifically looking for syringomyelia. As in infants, when an older child with CM II and hydrocephalus has undergone shunt placement the shunt must be thoroughly evaluated to ensure proper function.

\section{SURGICAL TREATMENT OF CM II}

Whether performed on an emergency basis in a stridorous infant or electively in a child with occipitocervical pain, decompression of the CM II can be a challenging undertaking and requires a thorough understanding of the

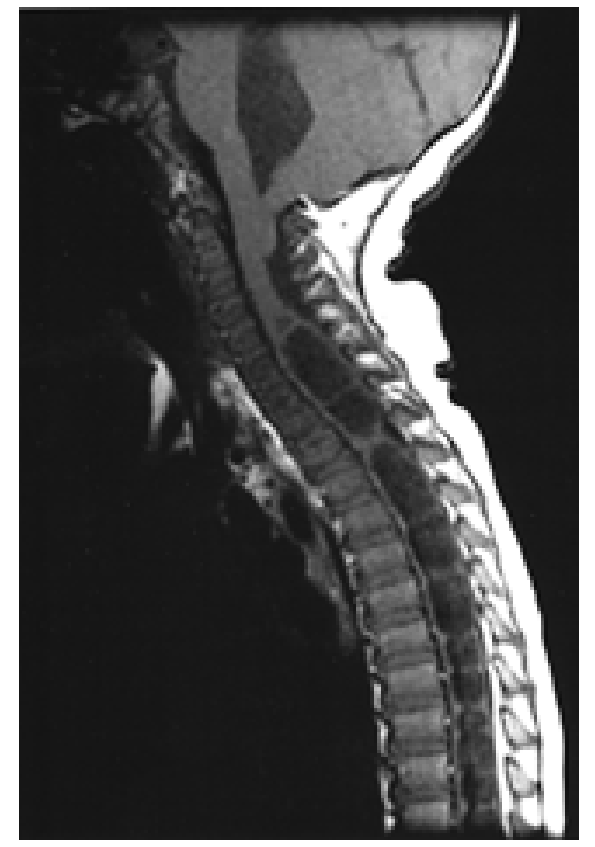

Fig. 7. Sagittal MR image obtained in a child with CM II and a large holocord syrinx.

abnormal anatomy to be encountered. The first step in preparing a child for surgery is ensuring that hydrocephalus is not the cause of the symptoms. In the child with a shunt, the shunt should be tapped and the function proven even in the presence of normal ventricular size. If any question exists as to the performance of the shunt, the device should be explored in the operating room prior to decompressing the CM II. In the child without a shunt, a thorough evaluation for latent hydrocephalus is performed and a shunt is placed if increased intracranial pressure exists.

Once hydrocephalus has been excluded as a factor in the production of symptoms, the patient is positioned prone on chest rolls. A horseshoe headrest is used for infants and a head-pin fixation device for older children to obtain flexion of the cervical spine. The skin incision is made to expose the entire pathological area: from the foramen magnum to the lowest level of cerebellar descent. The posterior cervical spine is exposed down through the avascular midline, avoiding the paraspinal musculature. The posterior elements of all spinal levels involved are exposed as well as the rim of the foramen magnum. The lateral exposure of the spine should stop short of the facets and should include the superior aspect of the first uninvolved level. Care should be taken exposing the posterior ring of $\mathrm{C}-1$ in infants because it is often incomplete and the dura with underlying brainstem is easily entered. Laminectomies of $\mathrm{C}-1$ through the lowest involved cervical segment are performed. It is arguably safer, and clearly more expeditious, to perform the laminectomies by using a high-speed air drill with footplate attachment. Suboccipital craniectomy is usually unnecessary, and often unwise, because the foramen magnum is typically larger than normal in CM II and does not often play a role in compression of the brainstem. The dura is now opened in the midline, exposing the malformation. Due to the gross- 
ly abnormal anatomy, several landmarks should be identified prior to intradural dissection. First, the caudal end of the cerebellum should be seen and the normal spinal cord directly caudal and ventral to it. If the caudal end of the cerebellum is not seen, extension of the dural opening or laminectomy of the next level may be necessary. Second, the choroid plexus is typically outside the fourth ventricle in its embryological position. The extraventricular choroid plexus can be followed into the fourth ventricle when this structure is not easily discernible. Third, the medullary kink, if present, must be identified. The malformed vermis and medulla can look strikingly similar, and great care should be taken in identifying the two structures. Finally, vascular structures, most commonly the posterior inferior cerebellar artery, can be present in the surgical field and should be sought out, identified, and protected. Identifying these structures is often difficult until arachnoid adhesions are dissected and the underlying structures are freed. If fourth ventricular outflow is seen, closure may begin. If CSF is not seen coming from the fourth ventricle, it is explored and its patency is confirmed. Arachnoid bands are often present and should be removed, usually with the aid of the operating microscope. Once CSF flow is established, the intradural space should be thoroughly irrigated with normal saline to wash out any blood and surgical debris to prevent intradural adhesions. A capacious dural patch graft is then sewn into place in a watertight fashion. The wound is closed in a standard manner. The resolution of symptoms should be expected within several days but can, on occasion, take weeks to improve.

In addition to the immediate risks of blood loss, infection, vascular and CNS injury, and persistence of symptoms, the patient with CM II faces other risks in a more delayed manner. Symptom recurrence after CM II decompression can be caused by shunt malfunction, inadequate initial decompression, bone regrowth after adequate decompression, ${ }^{13}$ epidural scarring with bandlike compression, syrinx formation, and cervical spondylolisthesis. Factors that have been found to be associated with the need for eventual reoperation include young age at initial decompression and failure to provide adequate CSF egress at the fourth ventricle. ${ }^{34}$ Cervical instability and kyphosis are well known delayed complications of cervical laminectomy in children. Radiographically evident instability of the upper cervical spine has been reported to be as high as $95 \%$ following CM II decompression, ${ }^{2}$ but a more recent report found the incidence of clinically significant instability to be much lower at $9 \% .{ }^{16}$ By limiting the laminectomies to the laminae themselves and avoiding disruption of the facet joints postoperative kyphosis and instability should be avoided.

\section{OUTCOME}

Despite the growing recognition of symptomatic CM II as a potentially life-threatening abnormality in children with myelomeningocele, up to $15 \%$ of patients with symptomatic CM II die by the age of 3 years and nearly one third are left with permanent neurological disability. ${ }^{18}$ When the subset of patients displaying symptoms of brainstem dysfunction is separated out, reported mortality rates after surgical decompression were as high as 50 to
$70 \%$ before a more aggressive surgical approach was taken. ${ }^{3,24}$ The high mortality rate was attributed to intrinsic brainstem dysmorphism and/or brainstem ischemia and hemorrhage. ${ }^{10}$ When a more urgent surgical course is taken, mortality rates in the literature improve to 15 to $23 \%,{ }^{28,40}$ suggesting that brainstem compression plays a reversible role in the production of life-threatening symptoms in symptomatic CM II. Mortality in older children primarily presenting with weakness and myelopathy should approach $0 \%{ }^{22,28}$

Prompt resolution of symptoms is the goal of surgical decompression, and reports in the literature support an aggressive surgical approach to symptomatic CM II. Good outcomes, as measured by resolution of preoperative symptoms, can be expected to approach $60 \%$ when the symptomatic infant is identified and decompression is expeditiously performed. ${ }^{28}$ Although good evidence exists that many infants with symptomatic CM II have dysplastic brainstems, ${ }^{10,12,35}$ Rauzzino and Oakes ${ }^{30}$ suggest that a dysplastic brainstem predisposes an infant to a rapid neurological decline when either compression or ischemia are added to the "brainstem at risk." Given the dismal outcomes in infants who do not undergo decompression, prompt surgical intervention appears warranted. Outcomes in older children presenting with myelopathy and/ or pain are much better, ranging from 79 to $100 \%$ improvement in symptoms. ${ }^{22,28}$

\section{Fetal Surgery and CM II}

Over the last several years, fetal intrauterine myelomeningocele repair has been a topic of hot debate. The body of literature on this technique has been growing, and a randomized controlled trial is currently underway. To date, intrauterine myelomeningocele repair has not been shown to improve lower extremity motor and sensory function, but there is a suggestion that this technique may reduce the severity of, or completely prevent, the $\mathrm{CM}$ II. ${ }^{36,37} \mathrm{In}$ addition, examples of both laboratory and clinical normalization of a preexisting CM II have been reported. ${ }^{4,38}$ This finding suggests that CM II is caused, at least in part, by the continuous flow of CSF through the neural placode. Tulipan ${ }^{36}$ theorizes that by interrupting this flow, the hindbrain can develop normally, and even reverse abnormal development. If randomized controlled trials provide evidence that intrauterine myelomeningocele repair reduces the incidence of hindbrain herniation, one of the largest paradigm shifts in the neurosurgical treatment of myelomeningocele will need to take place.

\section{CONCLUSIONS}

Despite more aggressive management of myelomeningocele, CM II continues to be the leading cause of death in infants and young children with open neural tube defects. Proper understanding of the embryology and anatomy of CM II is essential to understanding this complex feature of myelomeningocele. A proactive and aggressive approach to the potentially symptomatic child is warranted, and good outcomes can be expected when this course is taken. Intrauterine myelomeningocele repair has shown some preliminary promise, but as of now remains under investigation. So long as those who care for children with 
myelomeningocele continue to explore new approaches to often difficult management problems, the child born today with an open neural tube defect can expect the chance at a meaningful and productive life.

\section{References}

1. Arnold J: Myelocyste, Transposition von Gewebskeimen und Sympodie. Beitr Path Anat 16:1-28, 1894 (Reference unverified)

2. Aronson DD, Kahn RH, Canady A, et al: Instability of the cervical spine after decompression in patients who have ArnoldChiari malformation. J Bone Joint Surg Am 73:898-906, 1991

3. Bell WO, Charney EB, Bruce DA, et al: Symptomatic ArnoldChiari malformation: review of experience with 22 cases. J Neurosurg 66:812-816, 1987

4. Bouchard S, Davey MG, Rintoul NE, et al: Correction of hindbrain herniation and anatomy of the vermis after in utero repair of myelomeningocele in sheep. J Pediatr Surg 38:451-458, 2003

5. Cameron AH: The Arnold-Chiari malformation and other neuro-anatomical malformations associated with spina bifida. J Pathol Bacteriol 73:195-211, 1957 (Reference unverified)

6. Chiari H: Uber Veränderungen des Kleinhirns infolge von Hydrocephalie des Grosshirns. Dtsch Med Wschr 17:1172-1175, 1891

7. Cleland J: Contribution to the study of spina bifida, encephalocele, and anencephalus. J Anat Physiol 17:257-291, 1883 (Reference unverified)

8. Curnes JT, Oakes WJ, Boyko OB: MR imaging of hindbrain deformity in Chiari II patients with and without symptoms of brainstem compression. AJNR 10:293-302, 1989

9. el Gammal T, Mark EK, Brooks BS: MR imaging of Chiari II malformation. AJR 150:163-170, 1988

10. Gilbert JN, Jones KL, Rorke LB, et al: Central nervous system anomalies associated with meningomyelocele, hydrocephalus, and the Arnold-Chiari malformation: reappraisal of theories regarding the pathogenesis of posterior neural tube closure defects. Neurosurgery 18:559-564, 1986

11. Goldstein F, Kepes JJ: The role of traction in the development of the Arnold-Chiari malformation. An experimental study. J Neuropathol Exp Neurol 25:654-666, 1966

12. Holinger PC, Holinger LD, Reichert TJ, et al: Respiratory obstruction and apnea in infants with bilateral abductor vocal cord paralysis, meningomyelocele, hydrocephalus, and Arnold-Chiari malformation. J Pediatr 92:368-373, 1978

13. Hudgins RJ, Boydston WR: Bone regrowth and recurrence of symptoms following decompression in the infant with Chiari II malformation. Pediatr Neurosurg 23:323-327, 1995

14. Iskandar BJ, Oakes WJ: Chiari malformations, in Albright L, Pollack I, Adelson D (eds): Principles and Practice of Pediatric Neurosurgery. New York: Thieme, 1999, pp 165-187

15. Marin-Padilla M, Marin-Padilla TM: Morphogenesis of experimentally induced Arnold-Chiari malformation. J Neurol Sci 50:29-55, 1981

16. McLaughlin MR, Wahlig JB, Pollack IF: Incidence of postlaminectomy kyphosis after Chiari decompression. Spine 22: 613-617, 1997

17. McLendon RE, Crain BJ, Oakes WJ, et al: Cerebral polygyria in the Chiari Type II (Arnold-Chiari) malformation. Clin Neuropathol 4:200-205, 1985

18. McLone DG: Continuing concepts in the management of spina bifida. Pediatr Neurosurg 18:254-256, 1992

19. McLone DG, Knepper PA: The cause of Chiari II malformation: a unified theory. Pediatr Neurosci 15:1-12, 1989

20. McLone DG, Nakahara S, Knepper PA: Chiari II malformation: pathogenesis and dynamics. Concepts Pediatr Neurosurg 11: $1-17,1991$

21. Naidich TP, Pudlowski RM, Naidich JB, et al: Computed tomographic signs of the Chiari II malformation. Part I: skull and dural partitions. Radiology 134:65-71, 1980
22. Oakes WJ, Worley G, Spock A, et al: Surgical intervention in twenty-nine patients with symptomatic Type II Chiari malformations: clinical presentation and outcome. Concepts Pediatr Neurosurg 8:76-85, 1988

23. Padget DH: Development of so-called dysraphism; with embryologic evidence of clinical Arnold-Chiari and Dandy-Walker malformations. Johns Hopkins Med J 130:127-165, 1972

24. Park TS, Hoffman HJ, Hendrick EB, et al: Experience with surgical decompression of the Arnold-Chiari malformation in young infants with myelomeningocele. Neurosurgery 13: 147-152, 1983

25. Peach B: Arnold-Chiari malformation: anatomic features of 20 cases. Arch Neurol 12:613-621, 1965

26. Penfield W, Coburn DF: Arnold-Chiari malformation and its operative treatment. Arch Neurol Psychiatry 40:328-336, 1938 (Reference unverified)

27. Pollack IF, Kinnunen D, Albright AL: The effect of early craniocervical decompression on functional outcome in neonates and young infants with myelodysplasia and symptomatic Chiari II malformations: results from a prospective series. Neurosurgery 38:703-710, 1996

28. Pollack IF, Pang D, Albright AL, et al: Outcome following hindbrain decompression of symptomatic Chiari malformations in children previously treated with myelomeningocele closure and shunts. J Neurosurg 77:881-888, 1992

29. Pollack IF, Pang D, Kocoshis S, et al: Neurogenic dysphagia resulting from Chiari malformations. Neurosurgery 30: 709-719, 1992

30. Rauzzino M, Oakes WJ: Chiari II malformation and syringomyelia. Neurosurg Clin N Am 6:293-309, 1995

31. Rekate HL: To shunt or not to shunt: hydrocephalus and dysraphism. Clin Neurosurg 32:593-607, 1984

32. Ruge JR, Masciopinto J, Storrs BB, et al: Anatomical progession of the Chiari II malformation. Childs Nerv Syst 8:86-91, 1992

33. Russell DS, Donald C: The mechanism of internal hydrocephalus in spina bifida. Brain 58:203-215, 1935 (Reference unverified)

34. Sacco D, Scott RM: Reoperation for Chiari malformations. Pediatr Neurosurg 39:171-178, 2003

35. Sieben RL, Hamida MB, Shulman K: Multiple cranial nerve deficits associated with the Arnold-Chiari malformation. Neurology 21:673-681, 1971

36. Tulipan N: Intrauterine myelomeningocele repair. Clin Perinatol 30:521-530, 2003

37. Tulipan N, Hernanz-Schulman M, Bruner JP: Reduced hindbrain herniation after intrauterine myelomeningocele repair: a report of four cases. Pediatr Neurosurg 29:274-278, 1998

38. Tulipan N, Hernanz-Schulman M, Lowe LH, et al: Intrauterine myelomeningocele repair reverses preexisting hindbrain herniation. Pediatr Neurosurg 31:137-142, 1999

39. Van den Hof MC, Nicolaides KH, Campbell J, et al: Evaluation of the lemon and banana signs in one hundred thirty fetuses with open spina bifida. Am J Obstet Gynecol 162:322-327, 1990

40. Vandertop WP, Asai A, Hoffman HJ, et al: Surgical decompression for symptomatic Chiari II malformation in neonates with myelomeningocele. J Neurosurg 77:541-544, 1992

41. Venes JL, Black KL, Latack JT: Preoperative evaluation and surgical management of the Arnold-Chiari II malformation. J Neurosurg 64:363-370, 1986

42. Welch K, Shillito J, Strand R, et al: Chiari I "malformations"an acquired disorder? J Neurosurg 55:604-609, 1981

43. Wolpert SM, Anderson M, Scott RM, et al: Chiari II malformation: MR imaging evaluation. AJR 149:1033-1042, 1987

Manuscript received November 15, 2003.

Accepted in final form January 13, 2004.

Address reprint requests to: Kevin L. Stevenson, M.D., Pediatric Neurosurgery Associates, 5455 Meridian Mark Road, Suite 540, Atlanta, Georgia 30342. email: kstevenson@pediatricneurosurgery. net. 